\title{
Propagation of mesons in asymmetric nuclear matter in a density dependent coupling model
}

\author{
R. Aguirre and A.L. De Paoli \\ Department of Physics, La Plata National University \\ C.C. 67 (1900) La Plata, Argentina
}

\begin{abstract}
We study the propagation of the light mesons $\sigma, \omega, \rho$, and $a_{0}(980)$ in dense hadronic matter in an extended derivative scalar coupling model. Within the scheme proposed it is possible to unambiguously define effective density-dependent couplings at the lagrangian level. We first apply the model to study asymmetric nuclear matter with fixed isospin asymmetry, and then we pay particular attention to hypermatter in $\beta$-equilibrium. The equation of state and the potential contribution to the symmetry coefficient arising from the mean field approximation are investigated.

PACS 21.30.Fe,21.65.+f,12.40.Yx,26.60.+c
\end{abstract}

\section{INTRODUCTION}

In recent years the medium dependence of the meson-baryon couplings has been object of speculation [1] 9 . This subject has been promoted by the successes of the so-called quantum hadrodynamics theory (QHD) [10], in the relativistic description of diverse nuclear phenomena.

The assumption of variable couplings in the mean field approximation (MFA) is founded on different grounds. It can be interpreted as the trace of the quark structure of hadrons [7 [9], or it can be viewed as a way to match effective lagrangians and free-space nucleon-nucleon interactions [1 6]. In any case assigning a variable behavior to the couplings seems to be an appropriate method to interpolate from one dynamical regime to another, using effective hadron field models. A similar meaning has been given to the in-medium meson masses, which have been related to the transition to the chiral regime 111. The so-called Brown-Rho scaling law qualitatively describes the behaviour of the hadronic masses in the proximity of the transition point. According to the scaling law, all hadronic masses decrease approximately at the same rate as the system approaches to the chiral phase transition (with exception of the pseudo-scalar meson masses). Applied to the light vector mesons this hypothesis could explain some experimental results, as for example the dilepton production rate in heavy ion collisions.

On the other hand, in certain purely hadronic models [12 13] the non-polynomial meson-nucleon interaction gives rise to effective, density-dependent coupling in the MFA 14 16. In this paper we propose an extension of the derivative scalar coupling model (DSCM) of [13], which preserves the charge symmetry and provides effective couplings for all the mesonic channels. The medium dependence comes through the mean value of the scalar $\sigma$ meson, evaluated at finite density and temperature. More general interactions could include non-linear vertices in terms of all the mesons considered, but we restrict here to the simplest case. This choice is similar to the approach of [3], where the functional dependence of the couplings includes only the product $\bar{\Psi} \gamma_{\mu} \Psi$, but not the scalar $\Psi \Psi$ or more involved nucleon field combinations. As stressed in this reference, the density variation of the couplings must be written as a Lorentz invariant functional of the considered fields, to obtain the correct Euler-Lagrange equations. Otherwise the so called "rearrangement" contribution is absent and the thermodynamical consistency is lost.

We use the model proposed here to investigate asymmetric nuclear matter at finite temperature, therefore we explicitly include the isovector mesons $\rho$ and $a_{0}(980)$.

The equation of state of asymmetric matter is an important input in astrophysical studies such as the cooling rate of neutron stars or the supernova collapse mechanism. Also it is of interest in the description of heavy-ion collisions, where experiences with radioactive beams are expected to provide new insights into the structure of dense matter with high degree of asymmetry. This situation has stimulated the interest in this issue, and many theoretical works have been done in the last years [4] 17 [25].

As an important case of asymmetric matter we consider hadronic matter in $\beta$-equilibrium. For this purpose we generalize the DSCM model to include the octet of baryons $n, \Lambda, \Sigma$, and $\Xi$. It is well known that the proton concentration is determinant in the cooling of neutron stars 26. 227, and this concentration is mainly determined by the isospin-dependent contribution to the equation of state.

It is also of interest to study the meson properties under these conditions since, for instance, it could carry information about transient states of matter during heavy-ion collisions. This subject has been poorly developed in the literature. We have evaluated the meson propagators in the relativistic random phase approximation (RRPA), including particle-antiparticle contributions, and we have extracted from them the effective meson masses. 
We organize this paper presenting the model in Sec. II, in Sec. III we discuss the bulk properties of symmetric nuclear matter at zero temperature, meanwhile the asymmetric nuclear matter equation of state is treated in Sec. IV. The properties of $\beta$-stable matter are considered in Sec. V], and the Feynman graphs contributing to the RRPA, the evaluation of the propagators and the behavior of mesons in the hadronic environment are presented in Sec. V1. We conclude with the discussion and summary in Sec. VII.

\section{THE MODIFIED DSCM}

In this section we present a relativistic model of hadronic fields inspired on the DSCM proposed by Zimanyi and Moszkowski [13]. The DSCM has been used to study nuclear many-body effects in several applications [28], to investigate neutron star properties [29], extended to include nucleon resonances [30] and hyperons [31], related to an effective quark description of hadronic properties [32], and generalized with a tensor coupling [33] in order to improve the spin-orbit splitting.

The DSCM has two important features which distinguish it from the QHD-I model of Ref. [10]. In first place it is non-renormalizable $a b$ initio and there is no immediate way to introduce vacuum corrections to the MFA to the ground state, although the main properties of nuclear matter are successfully described. In second place a residual interaction can be extracted beyond the lowest order solution, whose strength decreases monotonically as a function of the baryonic density [15, 16]. This fact ensures the ground state predominance at high density as assumed in QHD [10].

Since we are interested here in the description of asymmetric matter, besides the fields $\Psi^{a}$ for the nucleons, we include the isoscalar scalar $(\sigma)$ and isoscalar vector $\left(\omega_{\mu}\right)$ mesonic fields, and those corresponding to the $\rho$ isovector vector $\left(\rho_{\mu}^{A}\right)$ and the $a_{0}(980)$ isovector scalar $\left(\delta^{A}\right)$ mesons. We use greek, latin lowercase and latin uppercase indexes to denote Lorentz, baryon isospin and meson isospin components, respectively.

In its simplest version the DSCM [13] has a Yukawa type $\mathrm{N}-\omega$ coupling and a $\mathrm{N}-\sigma$ non-polynomic term. We modify the vertices allowing for two different mesons (one of them the scalar $\sigma$ ) to locally interact with a baryon:

$$
\begin{aligned}
\mathcal{L}_{D S C} & =\bar{\Psi}\left[i \not \partial-\frac{M-g_{d} \boldsymbol{\tau} \cdot \boldsymbol{\delta}+g_{w} \psi+g_{r} \boldsymbol{\tau} \cdot \boldsymbol{\phi} / 2}{1+g_{s} \sigma / M}\right] \Psi+\frac{1}{2}\left(\partial^{\mu} \sigma \partial_{\mu} \sigma-m_{s}^{2} \sigma^{2}\right)+\frac{1}{2}\left(\partial^{\mu} \boldsymbol{\delta} \partial_{\mu} \boldsymbol{\delta}-m_{d}^{2} \boldsymbol{\delta}^{2}\right) \\
- & \frac{1}{4} F^{\mu \nu} F_{\mu \nu}+\frac{1}{2} m_{w}^{2} \omega^{2}-\frac{1}{4} \boldsymbol{R}^{\mu \nu} \boldsymbol{R}_{\mu \nu}+\frac{1}{2} m_{r}^{2} \boldsymbol{\rho}^{2}
\end{aligned}
$$

where $\Psi(x)$ is the isospin multiplet nucleon field, $M$ is the averaged nucleon mass and $g_{s}, g_{d}, g_{v}$, and $g_{r}$ are adimensional coupling constants. As usual in QHD the ground state for homogeneous infinite matter is approximated by considering mesonic fields as classical quantities and assimilating them to effective nucleon properties. Thus we can separate the c-number contributions:

$$
\begin{aligned}
\sigma(x) & =\bar{\sigma}+s(x), \\
\delta^{A}(x) & =\bar{\delta} \delta^{3 A}+d^{A}(x), \\
\omega_{\mu}(x) & =\bar{\omega} \delta_{\mu 0}+w_{\mu}(x), \\
\rho_{\mu}^{A}(x) & =\bar{\rho} \delta_{\mu 0} \delta^{3 A}+r_{\mu}^{A}(x),
\end{aligned}
$$

where $\bar{\sigma}, \bar{\delta}, \bar{\omega}$, and $\bar{\rho}$ are classical mean field values and $s, d^{A}, w_{\mu}$, and $r_{\mu}^{A}$ are quantum fluctuations which are not included in the ground state. Expressions for the c-number contribution to meson fields can be obtained by taking statistical averaged Euler-Lagrange equations, and requiring self-consistency. In this way we obtain:

$$
\begin{aligned}
m_{s}^{2} \bar{\sigma} & =g_{s} \frac{<\bar{\Psi}\left(M-g_{d} \tau_{3} \bar{\delta}+g_{w} \gamma_{0} \bar{\omega}+\frac{1}{2} g_{r} \tau_{3} \gamma_{0} \bar{\rho}\right) \Psi>}{M N^{2}} \\
m_{d}^{2} \bar{\delta} & =g_{d} \frac{<\bar{\Psi} \tau_{3} \Psi>}{N} \\
m_{w}^{2} \bar{\omega} & =g_{w} \frac{<\Psi^{\dagger} \Psi>}{N} \\
m_{r}^{2} \bar{\rho} & =g_{r} \frac{<\Psi^{\dagger} \tau_{3} \Psi>}{N}
\end{aligned}
$$

where we have used $N=1+g_{s} \bar{\sigma} / M$. The expectation values must be evaluated with the ground state solution for the nucleon field, which depends on $\bar{\sigma}$ and $\bar{\delta}$ through the effective nucleon mass: 


$$
M_{i}^{*}=\frac{M-g_{d} I_{i} \bar{\delta}}{N}
$$

with $I_{i}=1,-1$ for protons and neutrons, respectively. The nucleon dispersion relation is also modified according to $\left(p_{0}-g_{w} \bar{\omega}-g_{r} I_{i} \bar{\rho} / 2\right)^{2}-p^{2}=M_{i}^{* 2}$.

In Eqs. (8) and (9) the terms between angular brackets represent the conserved baryon density and the isospin density, respectively.

A residual nucleon-meson interaction arises beyond the lowest order approximation 15 by inserting Eqs. (2)-(5) in the interaction term:

$$
\frac{M+\sum_{i} \Gamma_{i} \phi_{i}}{1+g_{s} \sigma / M}=\frac{M+\sum_{i} \Gamma_{i}\left(\bar{\phi}_{i}+\delta \phi_{i}\right)}{N\left(1+\frac{g_{s}}{N} s\right)} .
$$

In the expression above the symbol $\phi_{i}$ represents any one of the mesonic fields $\delta, \omega$, and $\rho$, which by virtue of Eqs.(3)-(5) splits into the classical mean value $\bar{\phi}_{i}$, and the quantum fluctuation $\delta \phi_{i}$. $\Gamma_{i}$ stands for the bare meson nucleon vertices: $\Gamma_{i}=-g_{d} \tau, g_{w} \gamma, g_{r} \tau \gamma / 2$, corresponding to the $a_{0}, \omega$, and $\rho$ mesons respectively. The right hand side of this equation is non-polynomic and can not be used to directly apply a diagrammatic expansion. Restricting to the physical regime for which quantum fluctuations are negligible compared to mean values, enables us to approximate:

$$
\frac{M+\sum_{i} \Gamma_{i} \phi_{i}}{1+g_{s} \sigma / M} \simeq M^{*}+\gamma_{0} \delta \varepsilon+\mathcal{L}_{\text {res }}
$$

with

$$
\begin{aligned}
\delta \varepsilon & =\sum_{\omega, \rho} \Gamma_{i}^{*} \bar{\phi}_{i}, \\
\mathcal{L}_{\text {res }} & =-g_{s}^{*} s+\sum_{i} \Gamma_{i}^{*}\left[\delta \phi_{i}-\frac{g_{s}}{N M}\left(\bar{\phi}_{i}+\delta \phi_{i}\right) s\right] .
\end{aligned}
$$

We have introduced the medium dependent vertices $\Gamma_{i}^{*}$, which are obtained from the bare ones by replacing the coupling constants $g_{d}, g_{w}$, and $g_{r}$ by effective couplings. The last ones are given by the relation $g_{d} / g_{d}^{*}=g_{w} / g_{w}^{*}=g_{r} / g_{r}^{*}=N$. Also we have used $g_{s} / g_{s}^{*}=N^{2}$.

The expansion proposed in Eq. (11) respects the organizational principle of nuclear effective field theories [34].

In this approximation, the residual interaction of Eq.113) arises besides the nucleon effective mass (10) and the contribution to the nucleon single particle energy (12). The interaction term $\mathcal{L}_{\text {res }}$ comprises a one meson-nucleon vertex, together with a two-meson exchange term. In all cases the vertex functions are medium dependent, $g_{s}^{*}\left(1+\sum_{i} \Gamma_{i} \bar{\phi}_{i} / M\right), \Gamma_{d}^{*}, \Gamma_{w}^{*}$, and $\Gamma_{r}^{*}$ for the one-meson case and $g_{s}^{*} \Gamma_{i} / M$ for the two-meson instance.

This linearized version can be used to study the quantum corrections beyond the mean field approximation.

Variable couplings are an expected feature of hadronic models, whenever the quark substructure becomes relevant [7 9]. Furthermore, density dependent couplings have been proposed as a key assumption in order to match relativistic nucleon potentials adjusted to scattering data, with hadronic field models [1]. This approach was initiated as a way to avoid involved Brueckner-Hartree-Fock calculations for finite systems, using one boson exchange potentials. Thus, the main purpose is to take advantage of the relative simplicity of the Hartree approach to the QHD models. The link between both schemes is established by requiring the equality of the nucleon self-energy in symmetric nuclear matter as evaluated in both formulations, and allowing QHD coupling constants to be density dependent.

In our treatment the effective couplings are unambiguously extracted from the lagrangian, once the MFA has been introduced. Thus in this scheme one has a well defined and invariant way to describe the medium influence on the couplings. Furthermore, the internal consistency of the approach is guaranteed.

Up to this point we have restricted the discussion only to protons and neutrons, however the introduction of hyperons is straightforward. A sum over different baryonic species must be considered in the lagrangian density and the vertices must be modified in order to take into account the isospin degeneracy of each one. Also, additional couplings between the mesons and every hyperon iso-multiplet must be introduced. A more detailed discussion will be given in Sec. V.

The energy density $E$ for infinite homogeneous hadronic matter, can be evaluated in the MFA by taking the statistical average of the energy momentum tensor: $E=<T^{00}>$. The thermodynamical pressure $P$ under the same conditions is obtained by averaging the trace of the spatial-spatial component of this tensor: $P=<\operatorname{Tr} T^{i j}>/ 3$. We include the corresponding equations for the sake of completeness: 


$$
\begin{aligned}
E & =\sum_{i=p, n} \frac{1}{(2 \pi)^{3}} \int_{0}^{\infty} d^{3} k E_{k i}\left[n_{F}\left(E_{k i}\right)+n_{F}\left(-E_{k i}\right)\right]+\frac{1}{2}\left(m_{s}^{2} \bar{\sigma}^{2}+m_{d}^{2} \bar{\delta}^{2}-m_{w}^{2} \bar{\omega}^{2}-m_{r}^{2} \bar{\rho}^{2}\right) \\
& +g_{w}^{*} \bar{\omega} n+\frac{1}{2} g_{r}^{*} \bar{\rho}\left(n_{p}-n_{n}\right), \\
P & =\sum_{i=p, n} \frac{1}{3(2 \pi)^{3}} \int_{0}^{\infty} d^{3} k \frac{k^{2}}{E_{k i}}\left[n_{F}\left(E_{k i}\right)+n_{F}\left(-E_{k i}\right)\right]-\frac{1}{2}\left(m_{s}^{2} \bar{\sigma}^{2}+m_{d}^{2} \bar{\delta}^{2}-m_{w}^{2} \bar{\omega}^{2}-m_{r}^{2} \bar{\rho}^{2}\right),
\end{aligned}
$$

with $E_{k i}=\sqrt{M_{i}^{* 2}+k^{2}}, M_{i}^{*}$ is given by Eq. (10), and

$$
n_{F}\left(z_{i}\right)=\frac{\Theta\left(z_{i}\right)}{1+e^{\beta\left(x_{i}-\mu_{i}\right)}}+\frac{\Theta\left(-z_{i}\right)}{1+e^{\beta\left(x_{i}+\mu_{i}\right)}},
$$

is the nucleon statistical occupation number, $x_{i}=z_{i}+g_{w} \bar{\omega}+I_{i} g_{r} \bar{\rho} / 2$ is the particle energy, $\mu_{i}$ is the chemical potential, and $\beta=1 / k_{B} T$. The chemical potential is related to the number density of the i-type particle through:

$$
n_{i}=\frac{1}{(2 \pi)^{3}} \int_{0}^{\infty} d^{3} k\left[n_{F}\left(E_{k i}\right)-n_{F}\left(-E_{k i}\right)\right]
$$

and finally $n=n_{p}+n_{n}$ is the total particle number density.

To evaluate Eqs. (14) and (15), one must fix the particle number densities $n_{p}, n_{n}$ and then simultaneously solve Eqs. (6)-(10), together with Eq. (16).

Another interesting quantity is the nuclear symmetry energy $E_{s}$ defined as:

$$
E_{s}=\left.\frac{1}{2} \frac{\partial^{2} E}{\partial \chi^{2}}\right|_{\chi=0}
$$

where $\chi=\left(n_{n}-n_{p}\right) / n$. This energy contains a purely kinetic term $T$, and a contribution $V_{s}$ coming from the isovector mesons only. The explicit expression for $E_{s}$ at zero temperature, including the $a_{0}$ meson contribution has been already derived, see for example [21]:

$$
\begin{aligned}
E_{s} & =T+V_{s}, \\
T & =\frac{1}{12} \sum_{i=p, n} \frac{p_{F_{i}}^{2}}{E_{F_{i}}}, \\
V_{s} & =g_{r}^{* 2} \frac{n_{0}}{8 m_{r}^{2}}-g_{d}^{* 2} \frac{n_{0} \sum_{i}\left(\frac{M_{i}^{*}}{E_{F_{i}}}\right)^{2}}{4\left(m_{d}^{2}+g_{d}^{* 2} A\right)},
\end{aligned}
$$

where $p_{F_{i}}$ is the Fermi momentum for the i-type particle, $E_{F_{i}}=\sqrt{p_{F_{i}}^{2}+M_{i}^{* 2}}$, and

$$
A=\sum_{i=p, n} \frac{3}{\pi^{2} E_{F_{i}}}\left[M_{i}^{* 2} p_{F_{i}}+\frac{p_{F_{i}}^{3}}{3}-M_{i}^{* 2} \ln \left(\frac{p_{F_{i}}+E_{F_{i}}}{M_{i}^{*}}\right)\right]
$$

\section{BULK PROPERTIES OF SYMMETRIC NUCLEAR MATTER AT T=0}

In the previous section we have presented the model, which contains several free parameters. The masses of the $a_{0}, \omega$ and $\rho$ mesons are taken at their physical values $m_{d}=984 \mathrm{MeV}, m_{w}=783 \mathrm{MeV}$, and $m_{r}=770 \mathrm{MeV}$ respectively. We adopt the accepted value for the $\sigma$ meson mass $m_{s}=550 \mathrm{MeV}$. There remains to determine the four coupling constants. We adjust them to reproduce the main bulk properties of symmetric nuclear matter: the saturation density $n_{0}=0.15 \mathrm{fm}^{-3}$, the binding energy $\varepsilon_{B}=-15$ $\mathrm{MeV}$, and the symmetry energy $E_{s}=32 \mathrm{MeV}$ at zero temperature and at normal density. Another quantity of physical interest is the isothermal compressibility $\kappa_{T}$, however the DSCM provides very good values for $\kappa_{T}$ without imposing any further condition. Therefore we have three physical conditions to fix the four coupling constants. Two of them, $g_{s}$ and $g_{w}$ are univocally determined to take the values $g_{s}=12.379, g_{w}=14.624$, whereas $g_{r}$ and $g_{d}$ are functionally dependent through Eqs. (17)-(19) evaluated at $n=n_{0}$. For our calculations we have selected two sets of couplings $\left(g_{r}, g_{d}\right)$, denoted by $A$ and $B$ : set $A=(11.583,0)$ and set $B=(15,6.538)$, which are shown in Fig. 1 . 


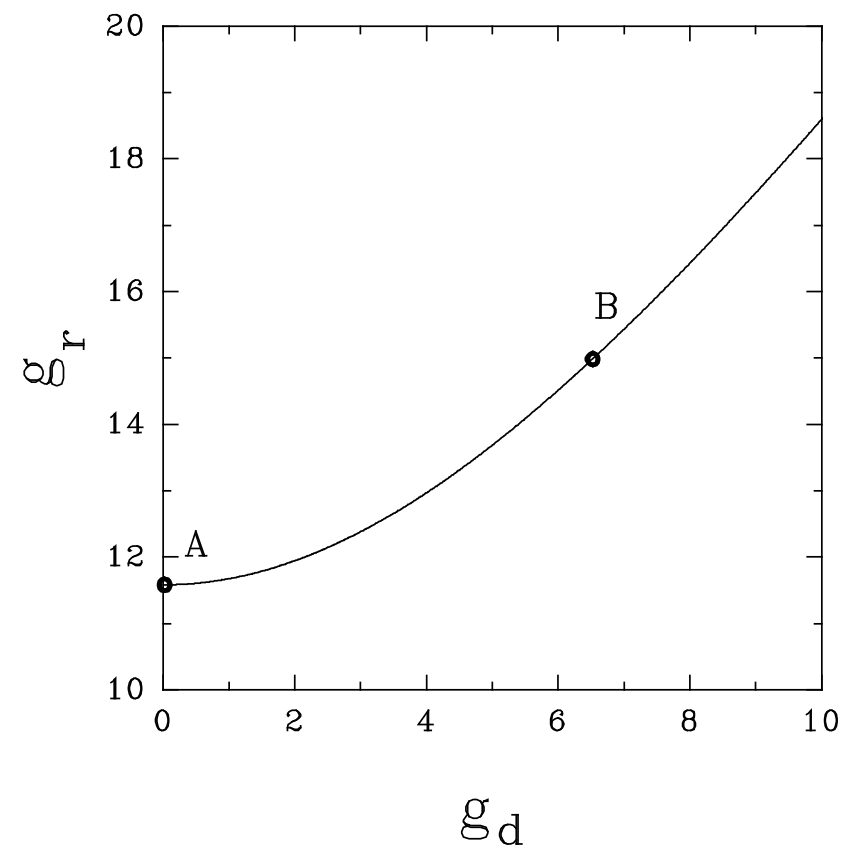

FIG. 1. The relationship between the couplings $g_{r}$ and $g_{d}$, constrained to reproduce the symmetry energy $E_{s}=32 \mathrm{MeV}$ at zero temperature and at the saturation density $n_{0}$. The pair of couplings $A$ and $B$ used in our calculations are marked with circles.

As previously mentioned, a feature of the model proposed is the presence of effective couplings. The behaviour of these couplings relative to their vacuum values is the same for the $\delta, \omega$, and $\rho$ fields, and different for the scalar $\sigma$, as discussed in Sec. II. As can be seen in Fig. 2, the channel corresponding to the last case is much more suppressed in dense matter. This figure corresponds to symmetric nuclear matter, but it must be noted that the behavior of the effective couplings depends on the composition of the hadronic medium, i.e. they must depend on the asymmetry coefficient $\chi$.

Although of diverse inspiration and derivation, we compare these results with the density dependent hadron field theory (DDHFT) outcomes [3 6 . F. For this purpose we use the interpolating algebraic function given in [5]. Differences are appreciable at medium and high densities. The couplings for $\sigma, \omega$, and $\rho$ are monotonous decreasing in both formalisms. A dropping of $20 \%$ for the isoscalar mesons, and of $42 \%$ for the $\rho$ meson is detected in the DDHFT at $n / n_{0}=2$. Our results provides for the same conditions a stronger decay of $60 \%$ for the $\sigma$ coupling and of $40 \%$ in the remaining cases.

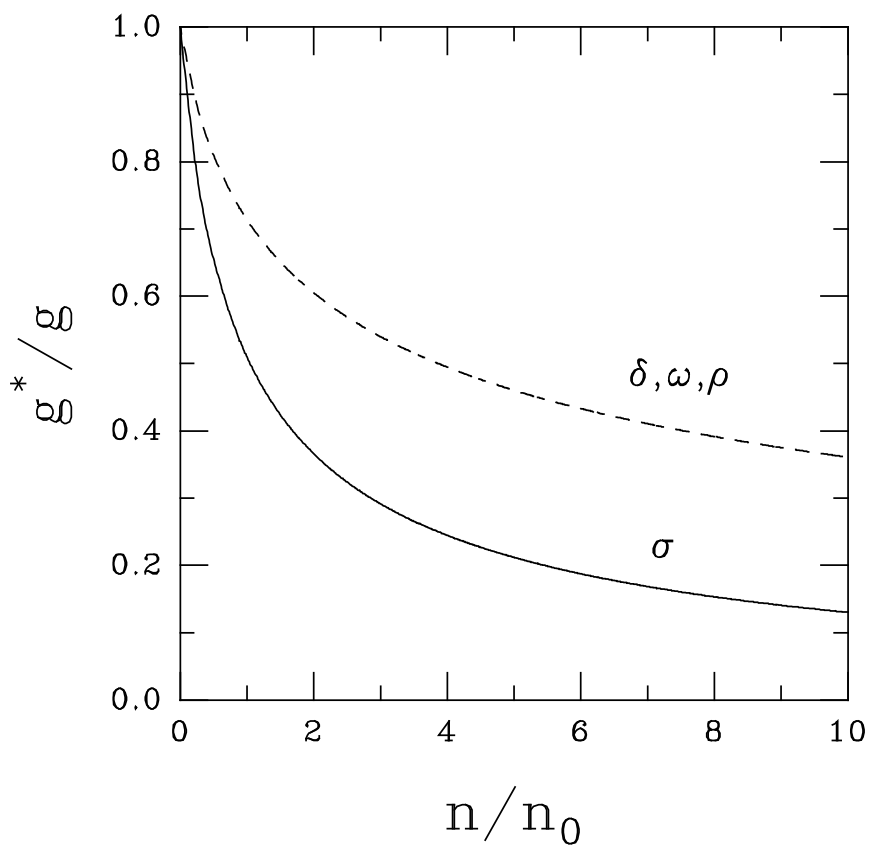

FIG. 2. The effective couplings relative to their vacuum values in symmetric nuclear matter at $\mathrm{T}=0$, in terms of the baryon density. The solid line corresponds to the $\sigma$ channel, and the dashed line to the $\delta, \omega$, and $\rho$ cases. 

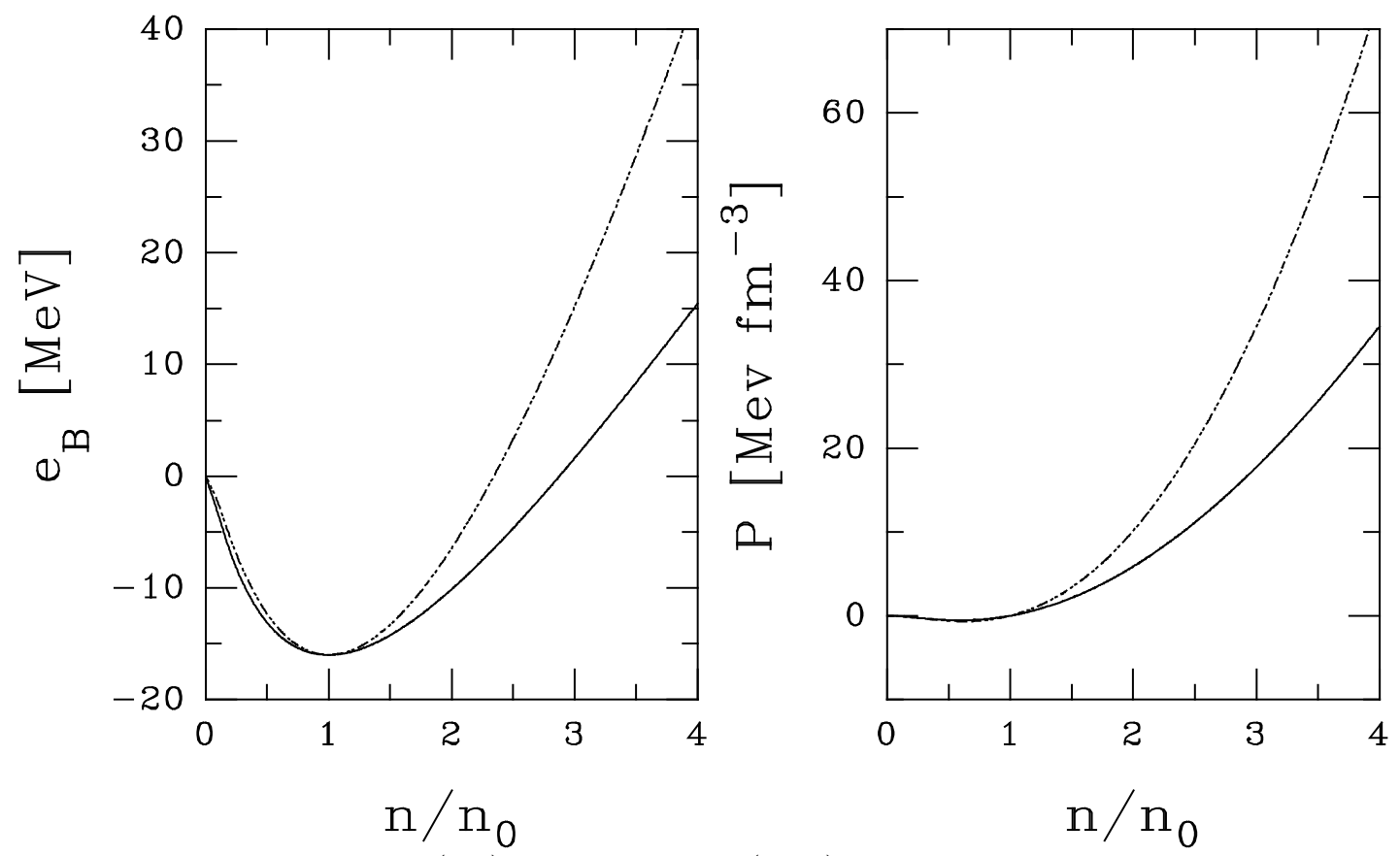

FIG. 3. The binding energy (left) and the pressure (right) as functions of the baryon number density at $\mathrm{T}=0$, in the MFA. Solid and dashed lines correspond to our results and the standard DSCM calculations, respectively.

With the sets of parameters $A$ and $B$ we have evaluated some properties of symmetric nuclear matter at zero temperature. In Fig. 3 we compare our results for the binding energy and the pressure as functions of the baryon number density, with the corresponding outcome of the standard DSCM. It can be seen that there are not appreciable differences below $n=1.5 n_{0}$, from here on both $\varepsilon_{B}$ and $P$ grow more slowly in our calculations. The isothermal compressibility is a measure of the stiffness of the pressure, we get at the saturation density $\kappa_{T}=165 \mathrm{MeV}$, against $\kappa_{T}=220 \mathrm{MeV}$ for the DSCM. The lower slope of the binding energy in our results is essentially due to the weakening of the repulsion at higher densities induced by the normalization factor $N$. On the other hand, the relative difference between the mean values $\bar{\sigma}$ and $\bar{\omega}$ increases with $n$ in our model, meanwhile in the standard DSCM it approaches to zero. This gives rise to the relative lessening of the pressure at high densities in our results.

The medium effects on the effective nucleon mass can be seen in Fig. 1, where a comparison with the DSCM result is made. In both cases $M^{*}$ is positive definite and monotonous decreasing, but the rate of falling at densities $0<n<2 n_{0}$ is more pronounced in our case because of the higher value of $g_{s}$ needed to reproduce the normal properties of nuclear matter. At higher values of the baryonic density $M^{*}$ stabilizes, due to the dynamical screening of the effective coupling.

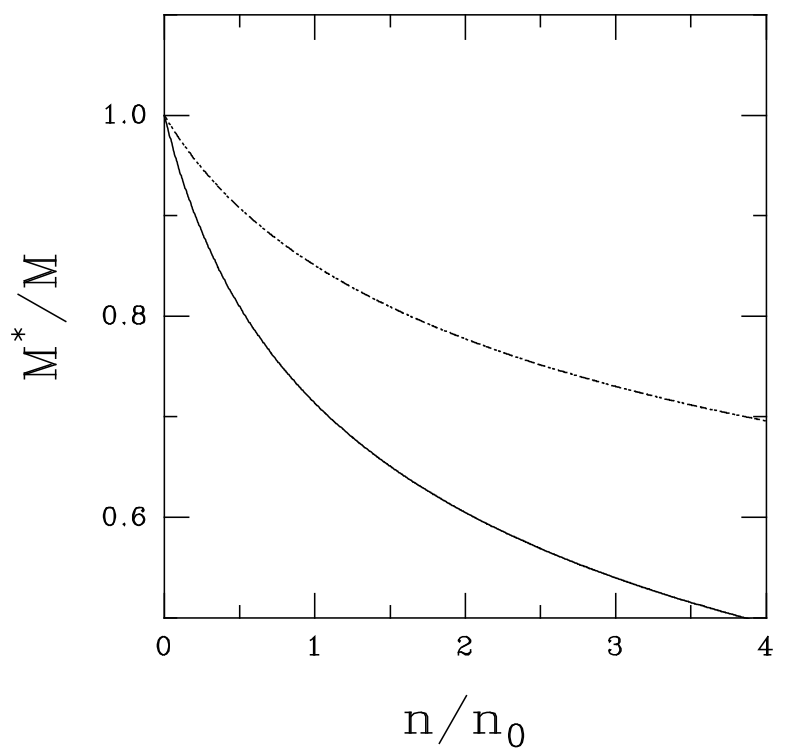

FIG. 4. The effective nucleon mass at zero temperature in symmetric nuclear matter. The line convention is the same as in Fig. 3 

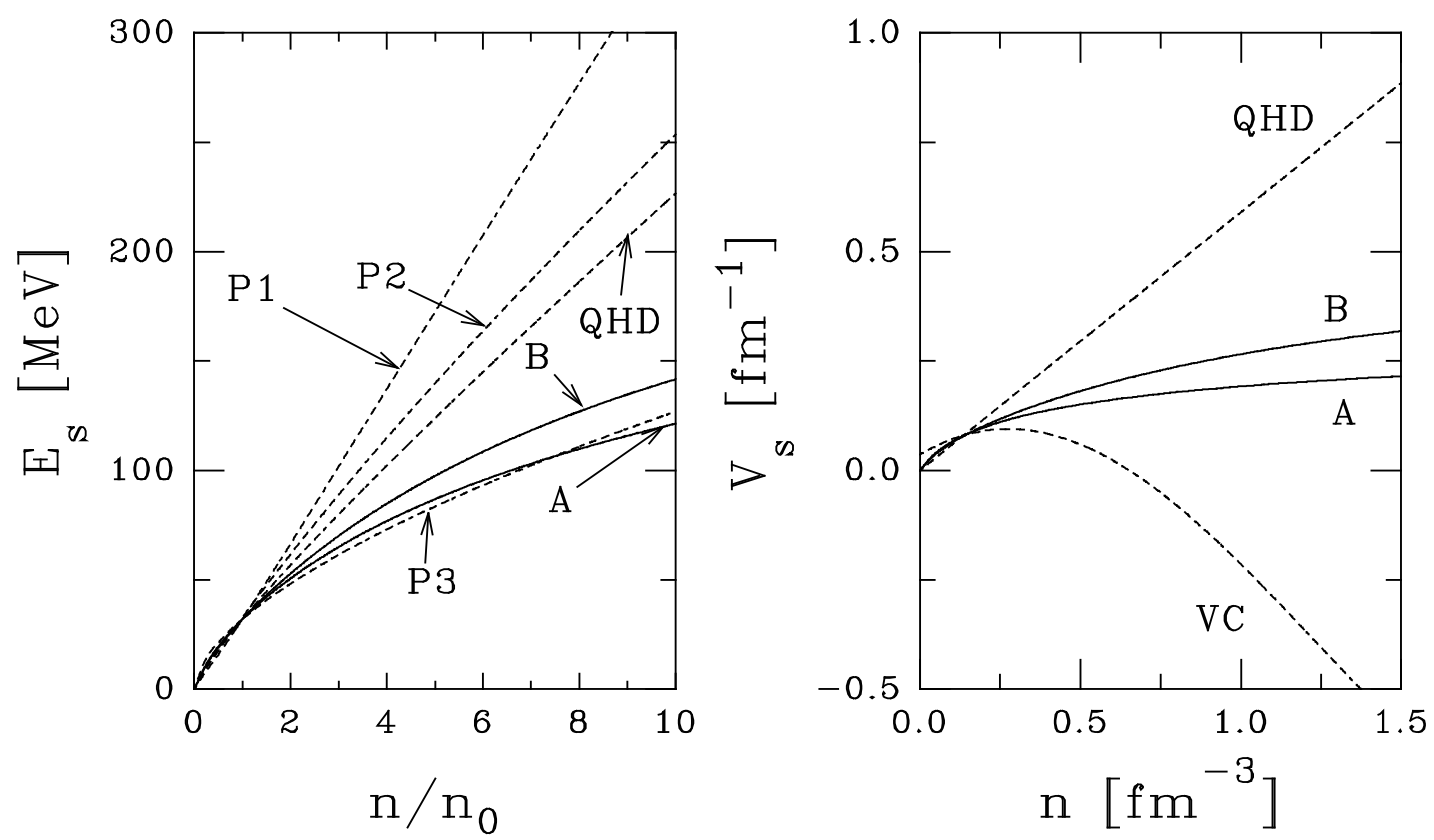

FIG. 5. The full symmetry energy (left panel) and the interaction contribution $V_{s}$ (right panel), in terms of the baryon number density. In both cases solid lines correspond to results with coupling sets A and B. Dashed lines are used in the left panel for the QHD-I model (without $\delta$ contribution), and the parameterizations P1, P2 and P3 as explained in the text. In the right panel dashed lines correspond to the QHD-I model and to the parameterization given in Ref. 22] for variational calculations (VC).

As the next step we investigate the density dependence of the symmetry energy $E_{s}$. It has been profusely studied in the past, using non-relativistic potentials as well as relativistic formulations 1723$]$. Recently $E_{s}$ has received attention by its applications to the study of the structure of nuclei with a large neutron excess, produced in the radioactive ion beam facilities. It is also a relevant subject in the evolution of neutron stars, determining the composition of the ground state and the cooling mechanism [26], or the phase transition to quark matter [22]. Furthermore, it has been proposed that the ratio of neutrons to protons in the pre-equilibrium stage of collisions between neutron rich nuclei could distinguish the asymmetric contribution of the nuclear equation of state [17].

Different theoretical predictions for $E_{s}$ produce rather dissimilar density dependences. In the left part of Fig. 5 we compare our results for the symmetry energy coefficient, with and without the contribution of the $a_{0}$-meson, with other commonly used descriptions. We include the result from the QHD-I model [10] without scalar-isovector coupling, and other three cases labeled P1, P2, and P3. The latter correspond to the phenomenological parameterizations [27]:

$$
\left.E_{s}=\frac{3}{5}\left(2^{2 / 3}-1\right) e_{F}\left[u^{2 / 3}-F(u)\right]+E_{s 0} F(u)\right]
$$

where $u=n / n_{0}, e_{F}$ is the non-relativistic Fermi energy at the saturation density, and the function $F(u)$ takes the forms $F_{1}(u)=2 u^{2} /(1+u), F_{2}(u)=u$, and $F_{3}(u)=\sqrt{u}$ for the curves denoted as P1, P2 and P3, respectively. All the curves are almost coincident for densities $n<1.5 n_{0}$, but their mutual differences become significant for densities above that limit. The exception corresponds to the cases A and P3, which differ each other only by negligible amounts in all the range of shown densities. From Eq. (19) it can be seen that the contributions to $V_{s}$ of the iso-vector $\delta$ and $\rho$ mesons are opposite in sign. However choosing $g_{d} \neq 0$ brings on an enhanced behaviour of $E_{s}$, because the value of $g_{r}$ required to adjust $E_{s}=32 \mathrm{MeV}$ at $n=n_{0}$ is bigger than $g_{d}$ (see Fig. 1). It must be noted that the rate of growth of the cases A, B, and P3 decreases with density, whereas it remains approximately constant for the curves $\mathrm{P} 1, \mathrm{P} 2$ and $\mathrm{QHD}-\mathrm{I}$.

The effect of polynomial self-interactions of the $\sigma$ field in QHD models has been studied in 25]. Both, the inclusion of exchange terms and of the $\delta$ coupling in Hartree approximation enhance the density dependence of $E_{s}$, and therefore diverge from curves $\mathrm{A}$ and B in the left part of Fig. 5 , being more alike to the P1 parametrization.

The behavior of $E_{s}$ depends on the method of evaluation and the model of interaction used, the latter defines the $V_{s}$ term. In the right part of Fig. . with the sets $\mathrm{A}$ and $\mathrm{B}$, and we compare them with $V_{s}$ extracted from the QHD-I model [10] and with the parameterization given by [22] for the variational calculations (VC) made in 35]. It can be seen that our results are intermediate between QHD-I and the VC results. A characteristic behaviour of the VC 
is that $V_{s}$ becomes negative for densities bigger than certain typical value, causing the disappearance of protons in neutron stars at high densities.

From the behavior of the symmetry terms shown in Fig. 5, we expect that the fraction of protons in star matter should be lower in our results as compared, for instance, with the QHD-I model prediction, although this fraction remains non vanishing for all densities in our case. The inclusion of the $\delta$ coupling (curve B) slightly increases the presence of protons.

\section{THE EQUATION OF STATE OF ASYMMETRIC NUCLEAR MATTER}

We study here the properties of nuclear matter at finite temperature by taking the asymmetry coefficient $\chi$ as a free parameter. In the next section the isospin asymmetry will be determined by the conditions of electric charge neutrality and matter stability against electroweak decay.

In first place we inspect the density dependence of the nucleon effective mass for fixed $\chi$. In Fig. 6 we compare results with and without $\delta$ coupling at $\mathrm{T}=0$ and $\chi=0.5$. For $g_{d}=0$ (set A) proton and neutron masses are degenerate, and for $g_{d} \neq 0$ (set B) the neutron (proton) mass is lowered (enhanced) due to medium effects. The splitting is heightened as the density increases.

Temperature effects are minimal in the range $0<T<100 \mathrm{MeV}$, and more noticeable at high densities. For example, when the coupling set $\mathrm{A}$ is chosen, an increment of about $5 \mathrm{MeV}$ in the nucleon effective mass is observed at $n=5 n_{0}$ as the temperature is raised from $T=0$ to $T=100 \mathrm{MeV}$, at a given $\chi$. Of the same magnitude but opposite in sign is the effect of increasing the asymmetry from $\chi=0$ to $\chi=1$ at a fixed temperature. When the coupling set B is used, it is found that the in-medium mass splitting $\Delta M^{*}=M_{p}^{*}-M_{n}^{*}$ decreases when the temperature is raised at fixed $\chi$. On the other hand, $\Delta M^{*}$ is enhanced when the asymmetry is isothermally increased. Numerical values of this mass splitting depend on the set of couplings used, and we estimate the magnitude of both temperature and asymmetry effects calculating $\Delta M^{*}$ with the set $\mathrm{B}$ at $n=5 n_{0}$. In this case the splitting reduces about $5 \mathrm{MeV}$ in neutron matter when the temperature covers the range $0<T<100 \mathrm{MeV}$, but an increment of approximately 50 $\mathrm{MeV}$ is found in $\Delta M^{*}$ if $\chi$ is varied between $\chi=0$ and $\chi=1$ at fixed temperature.

The thermodynamical pressure $P$ has been evaluated using Eq. (15), for several temperatures $0<$ $T<100 \mathrm{MeV}$, and several asymmetries $0<\chi<1$. As expected, increasing the temperature produces an enhancement of the pressure. This effect is strengthened by raising the asymmetry. The quantitative behavior of the pressure can be seen in Figs. 1 and 8 . In the first one we plot the pressure as a function of the number density at fixed asymmetry $\chi=0.25$ and for several temperatures. For $T \geq 20 \mathrm{MeV}$ it is a monotonous increasing function of the density, whereas for $T=0$ it exhibits a region of instability for densities below $n_{0}$. This instability gives rise to a liquid-gas phase transition [10]. The results in Fig. [7 correspond to the set A. By using the set B qualitatively similar results are obtained.

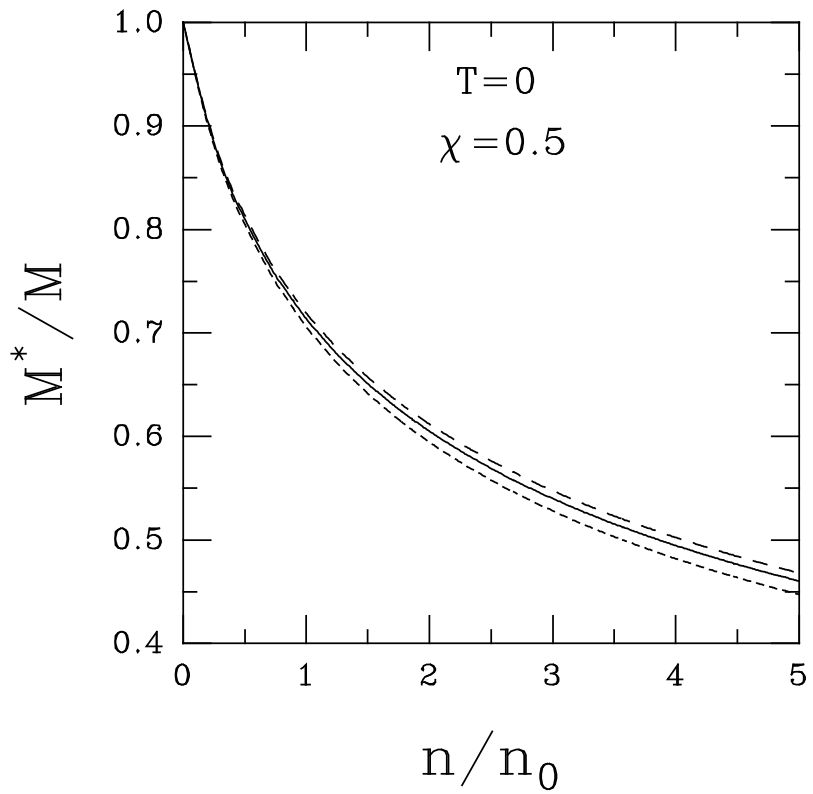

FIG. 6. The effective masses of proton and neutron as functions of the relative baryon number density for asymmetric nuclear matter at zero temperature. The solid (dashed) line corresponds to results with the coupling set $A(B)$. 


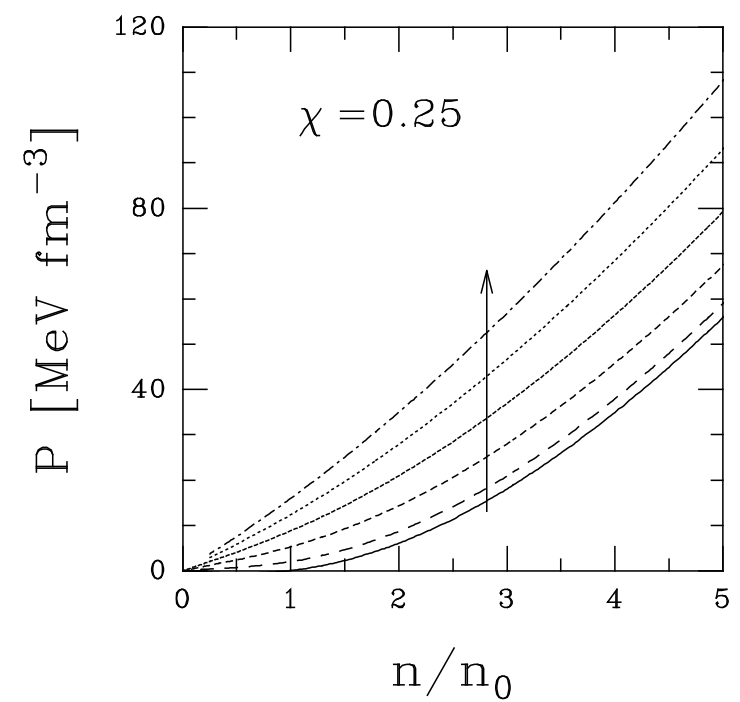

FIG. 7. The pressure $\mathrm{P}$ in terms of the nucleon number density at $\chi=0.25$. The different curves correspond to temperatures $T=0,20,40,60,80$, and $100 \mathrm{MeV}$. The arrow indicates the sense of growing temperatures. The results shown are obtained with the set $\mathrm{A}$.

The relevance of the asymmetry in our calculations can be observed in Fig. 8. The higher the values of the asymmetry the stiffer the pressure raises, this effect being emphasized when the coupling $g_{d}$ is non zero. The liquid-gas instability remains for low $T$ and $n$, disappearing for $\chi$ close to 1 .

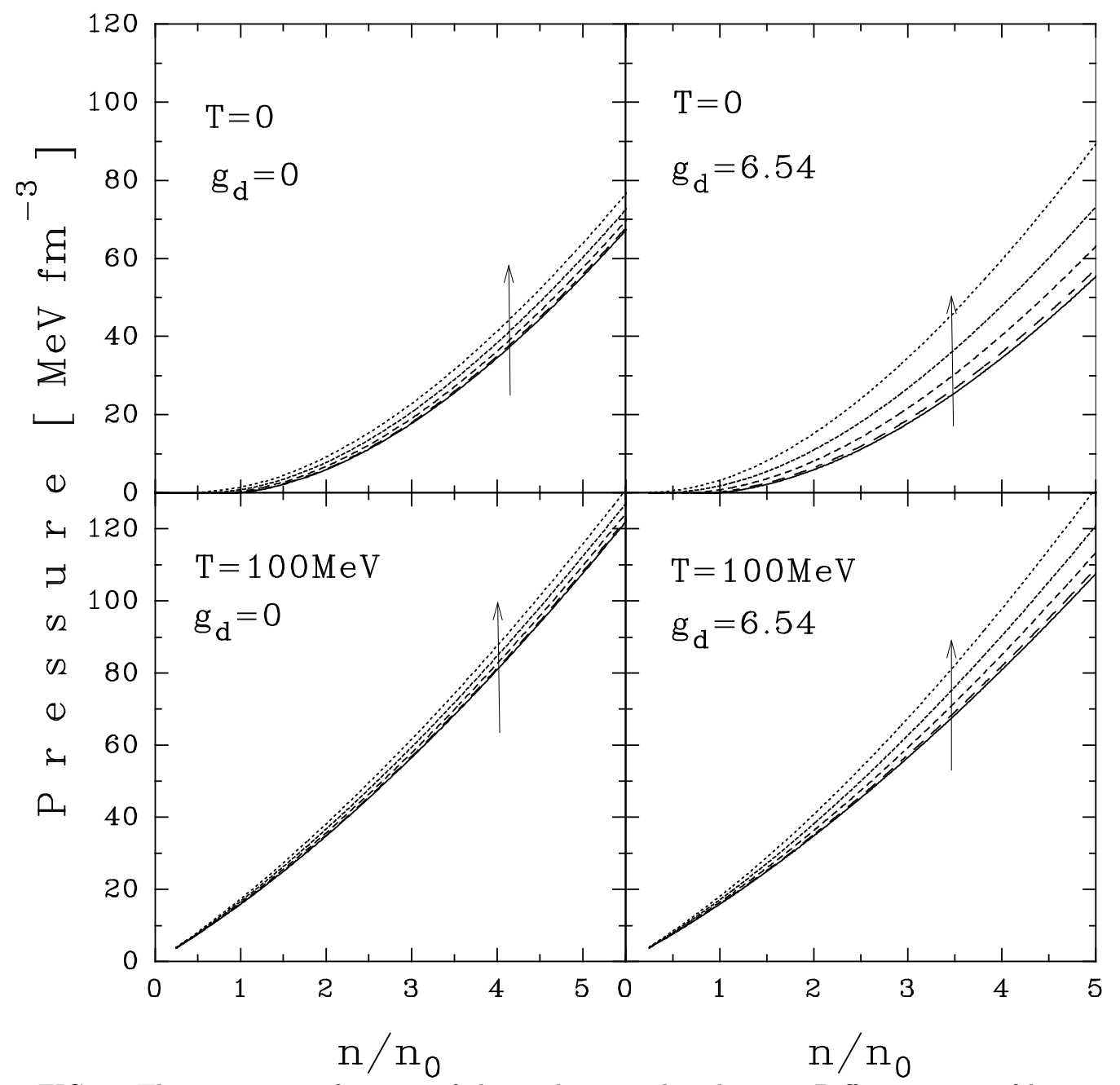

FIG. 8. The pressure as function of the nucleon number density. Different types of lines correspond to the asymmetry coefficients $\chi=0,0.25,0.50,0.75$, and 1.0 , respectively. The arrows indicate the direction of increasing $\chi$. The top (bottom) panel represents calculations for $\mathrm{T}=0(\mathrm{~T}=100 \mathrm{MeV})$, and the left (right) side is devoted to results using the coupling set A (B). 


\section{HADRONIC MATTER IN $\beta$-EQUILIBRIUM.}

The conditions in the interior of certain stellar objects like protoneutron stars, requires additional degrees of freedom to be included in the lagrangian density of Sec. [1]. Due to the large densities reached in such systems, several physical phenomena could take place. The appearance of mesons and baryons with strangeness, pion and/or kaon condensation, the chiral symmetry restoration, and the phase transition to a quark-gluon plasma are some of the expected processes. They must be taken into account, in order to properly describe the high density behavior of the equation of state. In this section we complete the model proposed by including the hyperons $\Lambda, \Sigma$, and $\Xi$, but we do not treat explicitly the chiral symmetry and quark degrees of freedom. Therefore our results should be valid until fluctuations preceding any phase transition become relevant. However we present here calculations in the range $0<n / n_{0}<10$ for the sake of comparison.

As anticipated in Sec. [1], the modifications in the lagrangian density are straightforward since we retain the form of the interaction for all the baryons. A sum over the full octet $N, \Lambda, \Sigma$, and $\Xi$ must be considered in Eq. (11), and new couplings $g_{s}, g_{d}, g_{w}$, and $g_{r}$ are introduced for the hyperons. Furthermore, the vertex between the $\rho$ meson and the baryon B must be modified by including an appropriate coefficient: $I_{B 3}=1 / 2$ for proton and $\Xi^{0}, I_{B 3}=-1 / 2$ for neutron and $\Xi^{-}, I_{B 3}=1$ for $\Sigma^{+}, I_{B 3}=0$ for $\Lambda$ and $\Sigma^{0}$, and $I_{B 3}=-1$ for $\Sigma^{-}$.

The new couplings should be fixed to reproduce some relevant quantity, according to the phenomenological approach. We proceed in this way to determine the $\sigma$ - and $\omega-\Lambda$ couplings. Using hypernuclei data the $\Lambda$ binding energy can be extrapolated to be $\varepsilon_{\Lambda}=-28 \mathrm{MeV}$ at $n_{0}$, thus we obtain $g_{s \Lambda}=2.335$, $g_{w \Lambda}=2.099$. For the other hyperons there are not accurate experimental data. Different arguments are commonly used to get numerical values, like $S U(6)$ symmetry or vector meson dominance. For simplicity and to carry out computations, we adopt $g_{s, w \Sigma}=g_{s, w \Xi}=g_{s, w \Lambda}$ and $g_{r, d \Sigma}=g_{r, d \Xi}=g_{r, d \Lambda}=g_{r, d}$, without any further justification. With this choice we obtain at $n_{0}$ very similar binding energies: $\varepsilon_{\Sigma}=-28.11$ $\mathrm{MeV}$, and $\varepsilon_{\Xi}=-28.27 \mathrm{MeV}$ for the $\Sigma$ and $\Xi$ hyperons.

Neutron star matter is electrically neutral, by additional contributions coming from electrons and muons. Leptons are included by means of Dirac free particle terms in the lagrangian of Eq. (1). The equilibrium for $\beta$ decay imposes constraints among the baryon and lepton chemical potentials: $\mu_{B}=$ $\mu_{n}-q_{B} \mu_{e}$. Here we have used $q_{B}$ for the baryon electric charge in units of the positron charge, $\mu_{n}, \mu_{e}$, and $\mu_{B}$ represents the chemical potentials for neutron, electron and the baryon $\mathrm{B}$, respectively. On the other hand, the electric charge neutrality imposes $0=-\sum_{l} n_{l}+\sum_{B} q_{B} n_{B}$, with $n_{l}$ and $n_{B}$ indicating the number density for leptons and baryons. At zero temperature we consider the Fermi momentum $p_{F}$, writing: $n_{i}=p_{F i}^{3} /\left(3 \pi^{2}\right), \mu_{l}=\sqrt{p_{F l}^{2}+m_{l}^{2}}$ for leptons, and $\mu_{B}=\sqrt{p_{F B}^{2}+M_{B}^{* 2}}+g_{w B}^{*} \bar{\omega}+g_{r}^{*} I_{B 3} \bar{\rho}$ for baryons. The efective mass $M_{B}^{*}$ is a generalization of eq. $(10), M_{B}^{*}=\left(M_{B}-I_{B} g_{d} \bar{\delta}\right) / N_{B}$ with $I_{B}=1$ for $p, \Sigma^{+}$, and $\Xi^{0}, I_{B}=0$ for $\Lambda$ and $\Sigma^{0}$, and $I_{B}=-1$ for $n, \Sigma^{-}$, and $\Xi^{-}$. We have used $N_{B}=1+g_{s B} \bar{\sigma} / M_{B}$, where $M_{B}$ is the averaged mass of the baryon isomultiplet B.

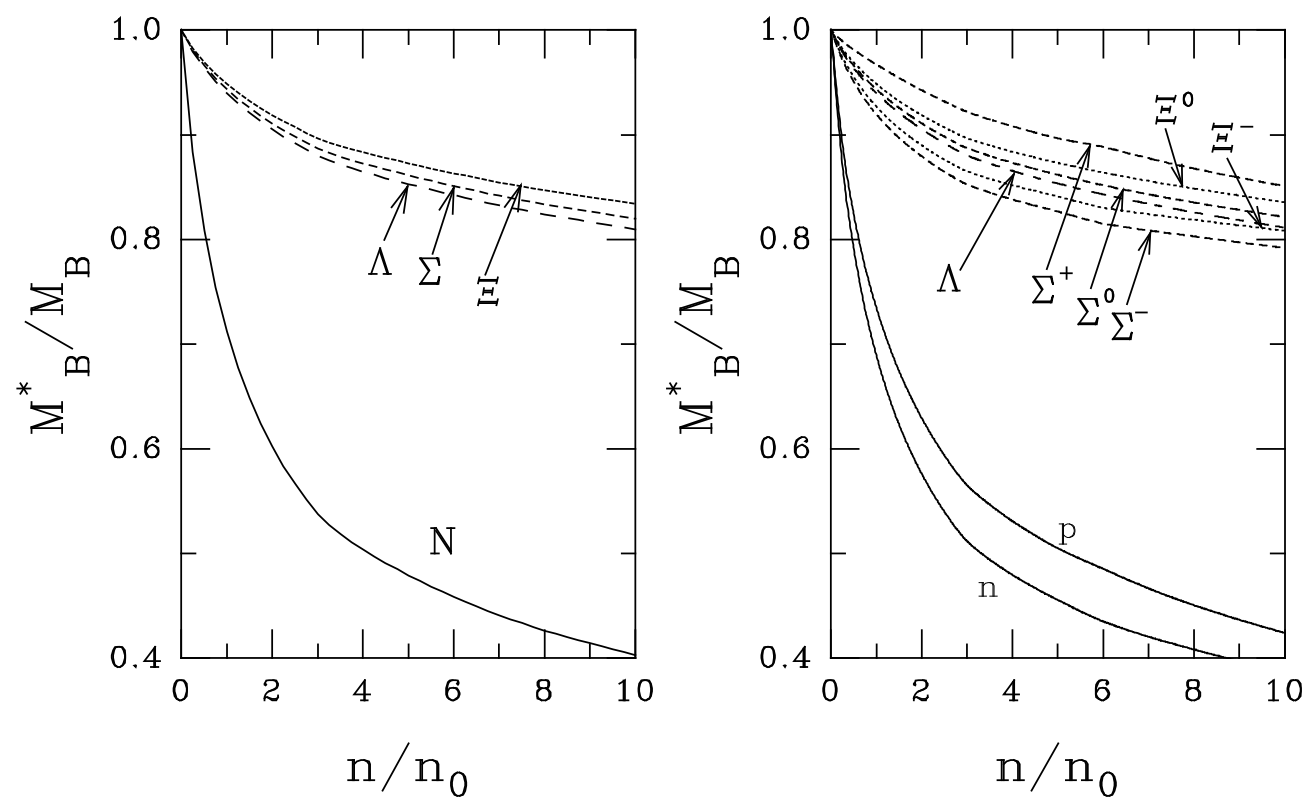

FIG. 9. The effective baryon masses in terms of the total baryon number density. The left (right) panel corresponds to calculations with set A (B). Different type of lines are assigned to each iso-multiplet, as indicated in both panels. 
The effective baryon masses as functions of the baryonic number density are shown in Fig. 9. In the results corresponding to set $\mathrm{A}$, each isomultiplet remains degenerate in mass. The variation of the hyperon masses are much more moderate than for the nucleon masses. As a consequence of the specific interaction used, the heavier the baryon considered, the weaker the density dependence of its effective mass is. Using the coupling set B the isospin degeneracy is removed, enhancing or dropping the mass of particles with positive or negative isospin projection, respectively, as it is depicted on the right part of Fig. 9 .

In Fig. 10 the relative population of the baryonic species is shown in terms of the total particle number, at $T=0$. In the range of densities studied the full baryon octet is present, with exception of the $\Xi^{0}$ when the coupling set B is used. The results obtained with $g_{d}=0$ and $g_{d}=6.538$ are very similar for the leptons and the lightest baryons (p, n, and $\Lambda$ ). Differences between them become noticeable in the growth of populations of the heavier fermions $\Sigma$ and $\Xi$. The more obvious is the early appearance, at $n \simeq 3.3 n_{0}$, and predominance of $\Sigma^{-}$particles in the results with the coupling set A.

The equilibrium baryonic population is not perturbed by the presence of the $\delta$ meson (set B) at low and medium densities. The effect of turning on the $\delta$ interaction, is twofold and is emphasized at high densities. In first place the baryon- $\delta$ interaction enhances the effective mass of $\Xi^{0}$ and diminishes that of $\Sigma^{-}$and $\Xi^{-}$, increasing and lowering the corresponding thresholds. The more evident consequence of this is the absence of $\Xi^{0}$ particles in the range $0<n / n_{0}<10$ (right panel of Fig. 10). In second place the coupling $g_{r}$ grows with $g_{d}$, affecting more strongly to the iso-triplet $\boldsymbol{\Sigma}$ than the iso-duplet $\boldsymbol{\Xi}$, due to the factor $I_{B 3}$. Since the $\rho$ meson contribution to the chemical potential is positive and greater for $\Sigma^{-}$ than for $\Xi^{-}$, this causes the appearance of $\Sigma^{-}$and of $\Xi^{-}$to be delayed and anticipated, respectively, going from the left to the right panel of Fig. 10. The $\Lambda$ and $\Sigma^{0}$ baryons, which do not couple to the isovector mesons, do not show appreciable changes in their distributions. On the other hand, from the comparatively earlier raising of the $\Sigma^{+}$and $\Xi^{-}$population obtained with set $\mathrm{B}$, it is possible to infer that, in absolute values, the $\delta$ contribution to the baryonic chemical potentials lies between one half and the total $\rho$ contribution. Of course, these results are partially a consequence of our assumption of equal couplings $g_{d}$ and $g_{r}$ for all the hyperons considered.
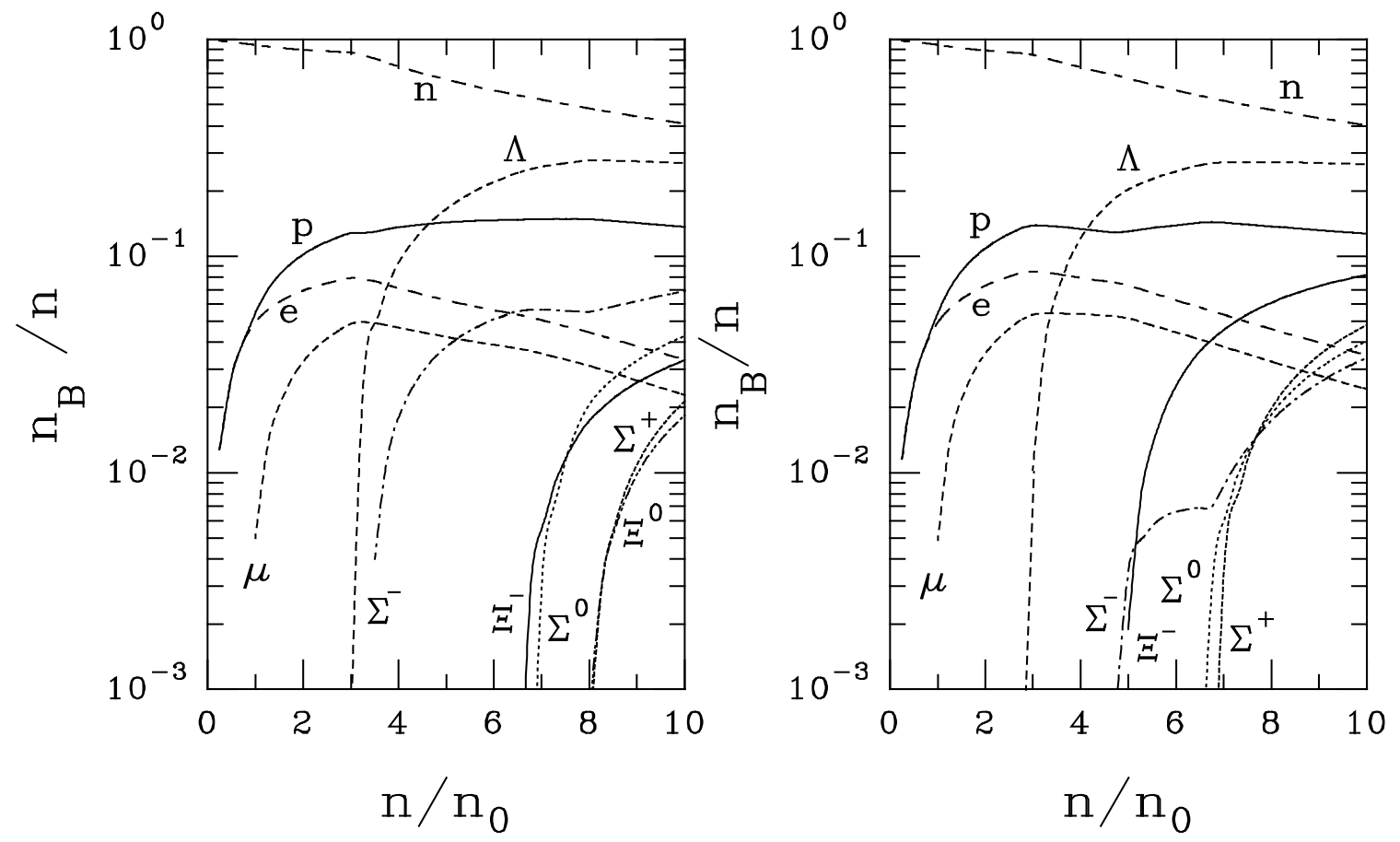

FIG. 10. The fraction of leptons and baryon species present in hadronic matter in $\beta$-equilibrium at $T=0$. The different curves are labeled with the corresponding particle name. The left (right) panel corresponds to calculations with the coupling set A (B).

The pressure in terms of the baryon number density is exhibited in Fig. 11. There are abrupt changes of slope in the curve corresponding to the set A, which coincide with the appearance of hyperons. Similar changes, but more attenuated, take place in the curve with the set B. 


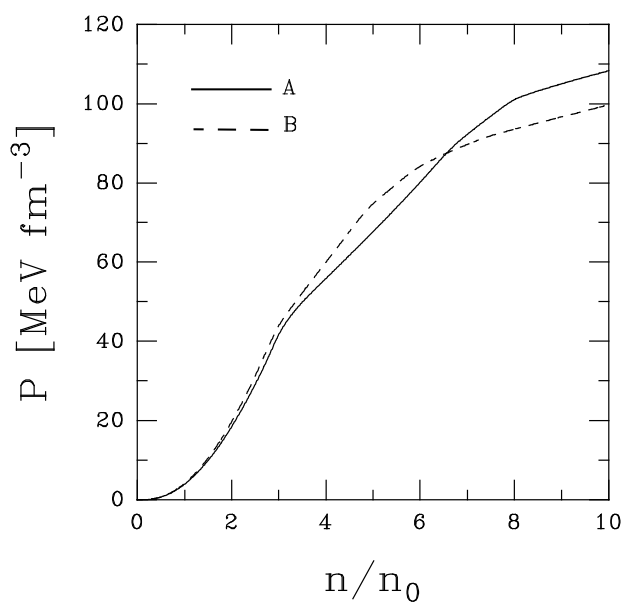

FIG. 11. The pressure of hadronic matter in $\beta$-equilibrium at $T=0$. As indicated in the figure, solid (dashed) line corresponds to calculations using the coupling set A (B).

\section{MESON PROPAGATION IN ASYMMETRIC NUCLEAR MATTER}

Medium effects in the meson properties have received attention in the later years, as they could carry the signals of phase transitions in the hadronic environment. As previously stressed, we expect our results to be valid out of the vicinity of the transition point.

In the MFA mesons are treated as classical fields, with constant mass. In order to include quantum corrections we must go beyond the MFA. This can be done in the relativistic random phase approximation (RRPA), using the linearized residual interaction of Eq. (13). In this approach the meson propagators are corrected by incorporating the baryon bubble diagrams at all orders, by using the Dyson-Schwinger equation. From the corrected propagator the effective meson mass can be extracted. This procedure has been applied in QHD calculations, see for example [10] and references listed therein. Specific computations with the DSCM can be found in [14,15].

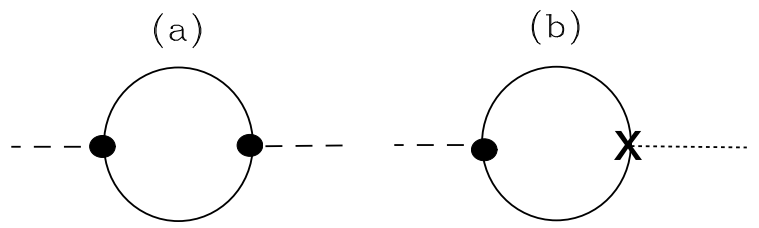

FIG. 12. Feynman diagrams included in the RRPA. Case a (b) corresponds to pure (mixing) meson propagation. The solid line stands for baryon propagator, dashed and dotted lines represent meson propagators of different types, and the filled circle and the cross their respective vertices.

At second order, the one-loop proper polarization insertions comprise the diagrams shown in Fig. 12 . The case (a) represent the propagation of a pure meson field, and the case (b) the mixing amplitude of different mesonic types. Due to baryon current conservation, the proper polarization for all the mesons can be written in terms of a few components. Some of them are divergent and requires an appropriate regularization. For this purpose, we follow the scheme outlined in 15. We do not deduce those results here, but we reproduce the main equations for the sake of completeness.

The formalism is best described within a generalized meson propagator, in a matrix representation of dimension equal to the sum of the mesonic degrees of freedom. For example the generalized free meson propagator $\mathcal{P}^{0}$, has in its diagonal blocks the free meson propagators $S^{0}(q), D^{0} A B(q), W_{\mu \nu}^{0}(q)$, and $R_{\mu \nu}^{0 A B}(q)$, for the $\sigma(x), \delta(x), \omega(x)$, and $\rho(x)$ fields, respectively, and null matrices in the complementary spaces:

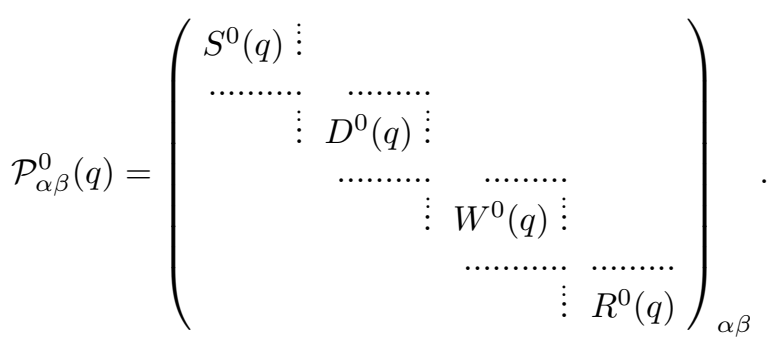


A similar expression holds for the full generalized propagator $\mathcal{P}$, but the complementary spaces are filled with the mixing meson propagators:

$$
\mathcal{P}_{\alpha \beta}(q)=\left(\begin{array}{cccc}
S(q) & M_{\sigma \delta}(q) & M_{\sigma \omega}(q) & M_{\sigma \rho}(q) \\
M_{\delta \sigma}(q) & D(q) & M_{\delta \omega}(q) & M_{\delta \rho}(q) \\
M_{\omega \sigma}(q) & M_{\omega \delta}(q) & W(q) & M_{\omega \rho}(q) \\
M_{\rho \sigma}(q) & M_{\rho \delta}(q) & M_{\rho \omega}(q) & R(q)
\end{array}\right)_{\alpha \beta} .
$$

The Dyson-Schwinger equation can be used to solve for $\mathcal{P}^{-1}(q)$ :

$$
\mathcal{P}_{\alpha \beta}^{-1}(q)=\mathcal{P}_{\alpha \beta}^{0-1}(q)-\Pi_{\alpha \beta}(q),
$$

where we have introduced the generalized polarization insertion

$$
\Pi_{\alpha \beta}(q)=\left(\begin{array}{cccc}
\Pi_{s}(q) & \Pi_{\sigma \delta}(q) & \Pi_{\sigma \omega}(q) & \Pi_{\sigma \rho}(q) \\
\Pi_{\delta \sigma}(q) & \Pi_{d}(q) & \Pi_{\delta \omega}(q) & \Pi_{\delta \rho}(q) \\
\Pi_{\omega \sigma}(q) & \Pi_{\omega \delta}(q) & \Pi_{w}(q) & M_{\omega \rho}(q) \\
\Pi_{\rho \sigma}(q) & \Pi_{\rho \delta}(q) & \Pi_{\rho \omega}(q) & \Pi_{r}(q)
\end{array}\right)_{\alpha \beta}
$$

Since we are primarily interested in the propagation of the pure meson fields, we do not consider mixing polarizations. The formulae for the one-loop diagonal components are as follows:

$$
\begin{aligned}
i \Pi_{s}(q) & =\sum_{B} g_{s B}^{* 2} \int \frac{d^{4} p}{(2 \pi)^{4}}\left\{\operatorname{Tr}\left[G_{B}(q) G_{B}(q+p)\right]+\sum_{\lambda} \frac{2 \bar{\phi}_{\lambda}}{M_{B}} \operatorname{Tr}\left[G_{B}(q) \Gamma_{\lambda} G_{B}(q+p)\right]\right. \\
& \left.+\sum_{\lambda, \lambda^{\prime}} \bar{\phi}_{\lambda} \bar{\phi}_{\lambda^{\prime}} \operatorname{Tr}\left[G_{B}(q) \Gamma_{\lambda} G_{B}(q+p) \Gamma_{\lambda^{\prime}}\right] / M_{B}^{2}\right\} \\
i \Pi_{d}^{A C}(q) & =g_{d}^{* 2} \sum_{B} \int \frac{d^{4} p}{(2 \pi)^{4}} \operatorname{Tr}\left[G_{B}(q) T^{A} G_{B}(q+p) T^{C}\right], \\
i \Pi_{w}^{\mu \nu}(q) & =\sum_{B} g_{w B}^{* 2} \int \frac{d^{4} p}{(2 \pi)^{4}} \operatorname{Tr}\left[G_{B}(q) \gamma^{\mu} G_{B}(q+p) \gamma^{\nu}\right], \\
i \Pi_{r \mu \nu}^{A C}(q) & =g_{r}^{* 2} \sum_{B} \int \frac{d^{4} p}{(2 \pi)^{4}} \operatorname{Tr}\left[G_{B}(q) \gamma_{\mu} T^{A} G_{B}(q+p) \gamma^{\nu} T^{C}\right]
\end{aligned}
$$

where the index $B$ runs over all the baryons considered, and $\lambda, \lambda^{\prime}$ in the first equation runs over the meson fields $\delta, \omega$, and $\rho$. The vertices $\Gamma_{\lambda}$ have been described in Sec. [1]. The baryon propagators $G_{B}(q)$ are evaluated in the MFA. In our calculations we only need the transversal component in the Lorentz indices, and the third component of isospin. For this purpose we use $T^{3}=\tau^{3}$ for the nucleon and $\Xi, T^{3}=1$ for the $\Lambda$, and $T^{3}=\operatorname{diag}(1,0,-1)$ for the $\Sigma$ particle. The referred expressions contain particle-antiparticle, particle-hole, and Pauli blocking contributions. The former one is divergent, to extract finite contributions we apply the regularization scheme outlined in 15]. The Lorentz scalar contributions, containing the integrand $\operatorname{Tr}\left[G_{B}(q) G_{B}(q+p)\right]$, remains undefined by a constant $\lambda$, related to the covariant derivative of the polarization evaluated at the regularization point. We take this constant as a free parameter to analyze the possible dynamical regimes. There are two independent parameters $\lambda_{s}$ and $\lambda_{d}$ corresponding to the diagonal components $\Pi_{s}$ and $\Pi_{d}$ respectively. We require null contribution for the polarization evaluated on the meson mass shell, at zero baryon density and temperature. Thus we obtain for the finite particle-antiparticle contribution of the baryon-B bubble:

$$
\begin{aligned}
& \Pi_{v B}^{\prime 00}(q)=\frac{g_{v B}^{* 2}}{2 \pi^{2}} q^{2} \int_{0}^{1} d z z(1-z) \ln \left[\frac{M_{B}^{* 2}-z(1-z) \mathrm{q}^{2}}{M_{B}^{2}-z(1-z) m_{v}^{2}}\right] \\
& \Pi_{v B}^{\prime 33}(q)=\frac{\mathrm{q}^{2}}{q^{2}} \Pi_{v B}^{\prime 00}(q), \\
& \Pi_{c B}^{\prime}(q)=\lambda_{c} \frac{g_{c B}^{* 2}}{8 \pi^{2}}\left(m_{B}^{* 2} m_{c}^{2}-\mathrm{q}^{2}\right)-\frac{3 g_{c B}^{* 2}}{4 \pi^{2}} \int_{0}^{1} d z\left[M_{B}^{* 2}-z(1-z) \mathrm{q}^{2}\right] \ln \left[\frac{M_{B}^{* 2}-z(1-z) \mathrm{q}^{2}}{M_{B}^{2}-z(1-z) m_{c}^{2}}\right],
\end{aligned}
$$



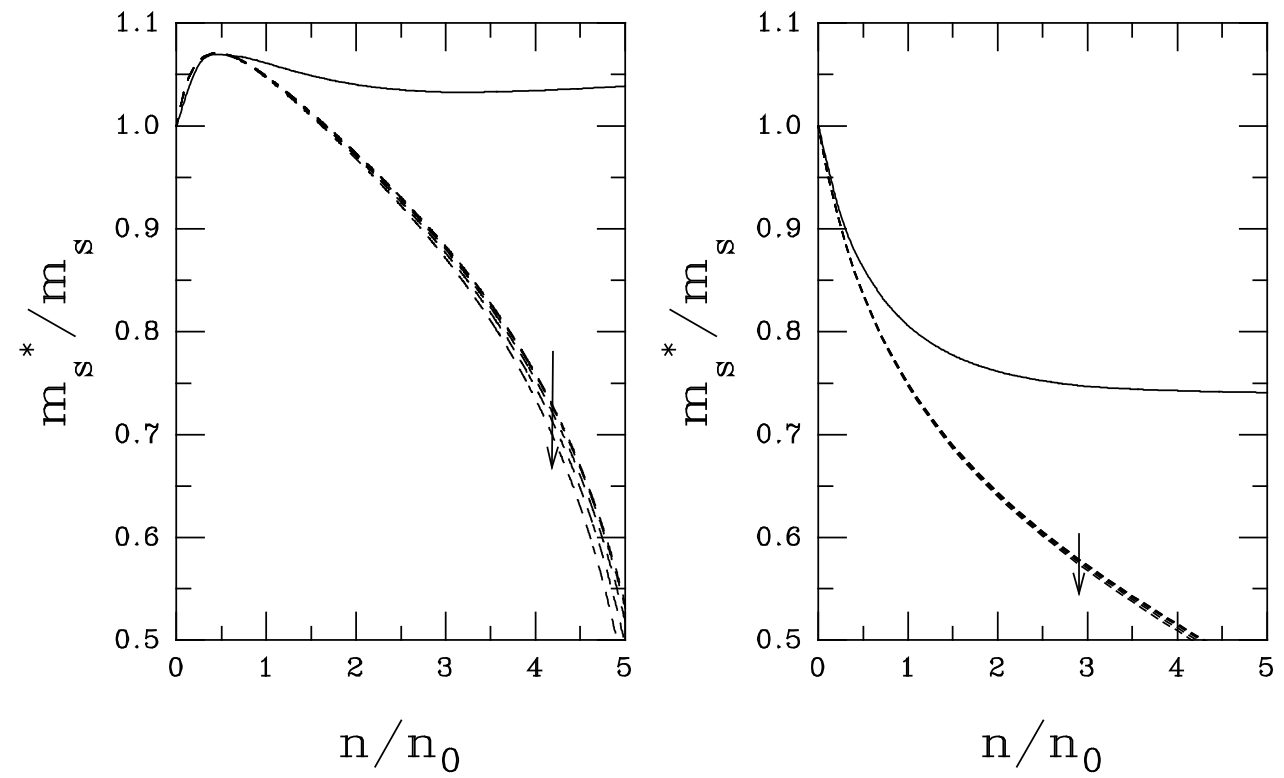

FIG. 13. The effective $\sigma$-meson mass as a function of the baryonic density at $\mathrm{T}=0$. In the left (right) panel the results for the regularization parameter $\lambda_{s}=10\left(\lambda_{s}=100\right)$ are plotted. In each case the solid line corresponds to hadronic matter in $\beta$-equilibrium, and the dashed lines to nuclear matter with asymmetry coefficients $\chi=0,0.25,0.50,0.75$, and 1 . The arrow indicates the direction of growing $\chi$ in the last case.

where the index $v=w, r$ runs over the vector mesons, and $c=s, d$ runs over the scalar ones. In the case of isovector polarizations, it must be regarded as the $(3,3)$ isospin component. Furthermore we have used $\mathrm{q}^{2}=q_{\mu} q^{\mu}, q$ is the modulus of the spatial component of the momentum, and $m_{B}^{*}=M_{B}^{*} / M_{B}$.

Once the polarization has been properly defined, we introduce the effective meson masses $m_{s}^{*}, m_{d}^{*}, m_{w}^{*}$, and $m_{r}^{*}$. They have been defined as the zeroes of the corresponding inverse propagators at zero vector momentum, i.e. the $p_{0}$ solutions of:

$$
\mathcal{P}_{a a}^{-1}\left(p_{0}, p=0\right)=\mathcal{P}_{a a}^{0-1}\left(p_{0}, p=0\right)-\Pi_{a a}\left(p_{0}, p=0\right)=0,
$$

for $a=s, d, w$, and $r$.

In Figs. 1315 we display the numerical results for the density dependence of the meson masses at $T=0$, under different compositions of the hadronic medium. In Fig. 13 the behavior of the $\sigma$ meson mass is presented. The results correspond to the coupling set A, there are no appreciable differences with respect to the calculations using set B .

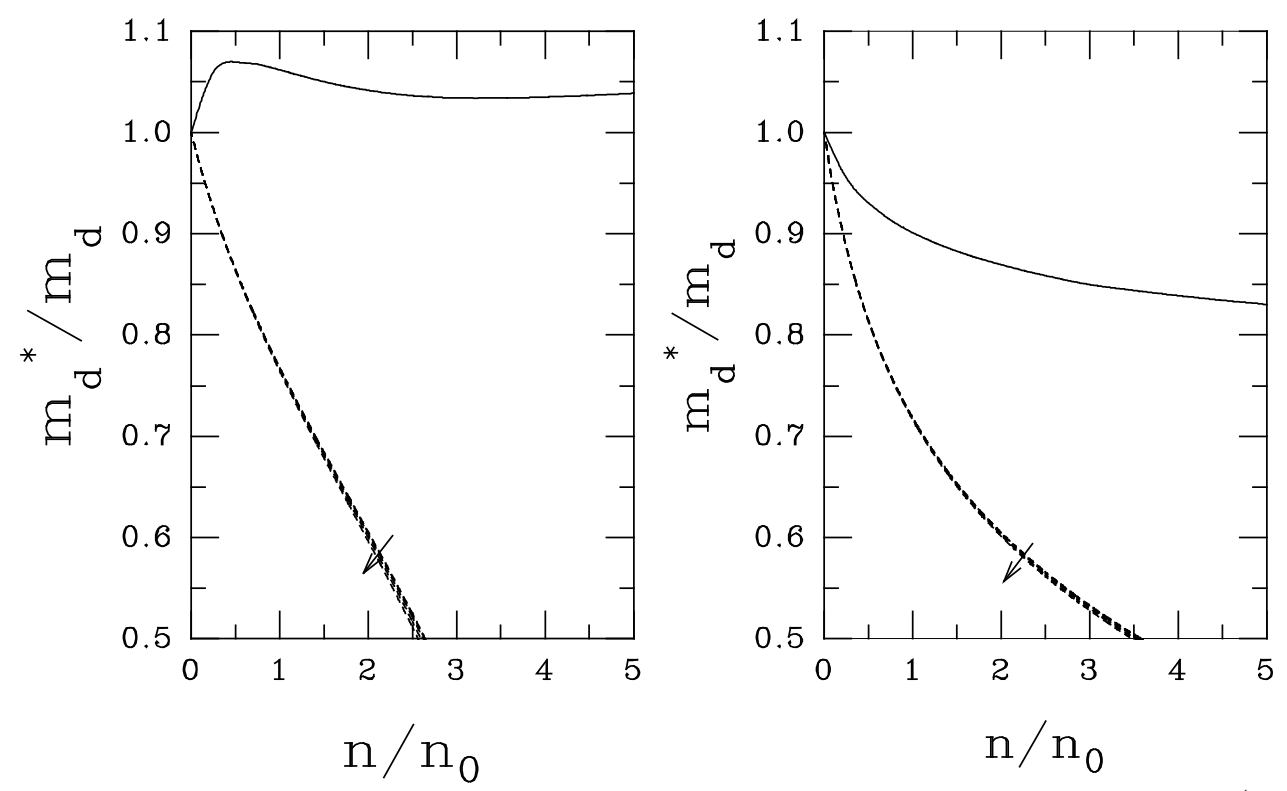

FIG. 14. The effective $a_{0}$-meson mass as a function of the density at $\mathrm{T}=0$. The left (right) panel corresponds to the values $\lambda_{d}=10\left(\lambda_{d}=100\right)$. Solid line stands for $\beta$-stable hadronic matter, and dashed lines correspond to asymmetric nuclear matter with asymmetry $\chi=0.25,0.50,0.75$, and 1 . The arrows indicate the direction of growing $\chi$. 
It can be seen that in $\beta$-stable hadronic matter the mass is almost constant at high densities. In the case of nuclear matter at constant asymmetry the density dependence is more pronounced, and monotonous decreasing. The asymmetry dependence is small, as this figure shows.

Fig. 14 is devoted to the $a_{0}$ meson mass. The curves for matter in $\beta$-equilibrium are qualitatively similar to those corresponding to the $\sigma$ meson. In asymmetric nuclear matter its behavior is much more striking. For $\lambda_{d}=10$ the mass becomes zero at $n / n_{0} \simeq 4$, whereas for $\lambda_{d}=100$ it decreases sharply but never vanishes in the whole range considered.

The masses for the vector mesons are shown in Fig. 15. For the $\omega$ meson the behavior is almost independent of the composition of the hadronic environment, sensible departures are observed only for extreme densities. This is not the case of the $\rho$ meson mass, a clear difference among $\beta$-stable matter and asymmetric nuclear matter is shown, even at low densities.

Since $\Pi_{a a}(p)$ receives the contribution of all the baryonic species considered, the mesonic effective masses are strongly influenced by the inclusion of hyperons, even at densities close to the normal saturation value. It must be noted that the particle-antiparticle term coming from the baryon-B bubble contributes even when this particle is not present on its Fermi shell. The hyperon particle-antiparticle contributions at medium and high densities causes the stabilization of the mesonic masses in neutral $\beta$-stable matter. This can be appreciated in Figs. 13 15 where they exhibit a weaker density dependence as compared to nuclear matter results. The magnitude of this effect is distinct for each type of meson, depending on the strength of its coupling to the hyperons. This fact explains why the omega-meson mass is little affected by the presence of hyperons relative to the rho-meson mass.
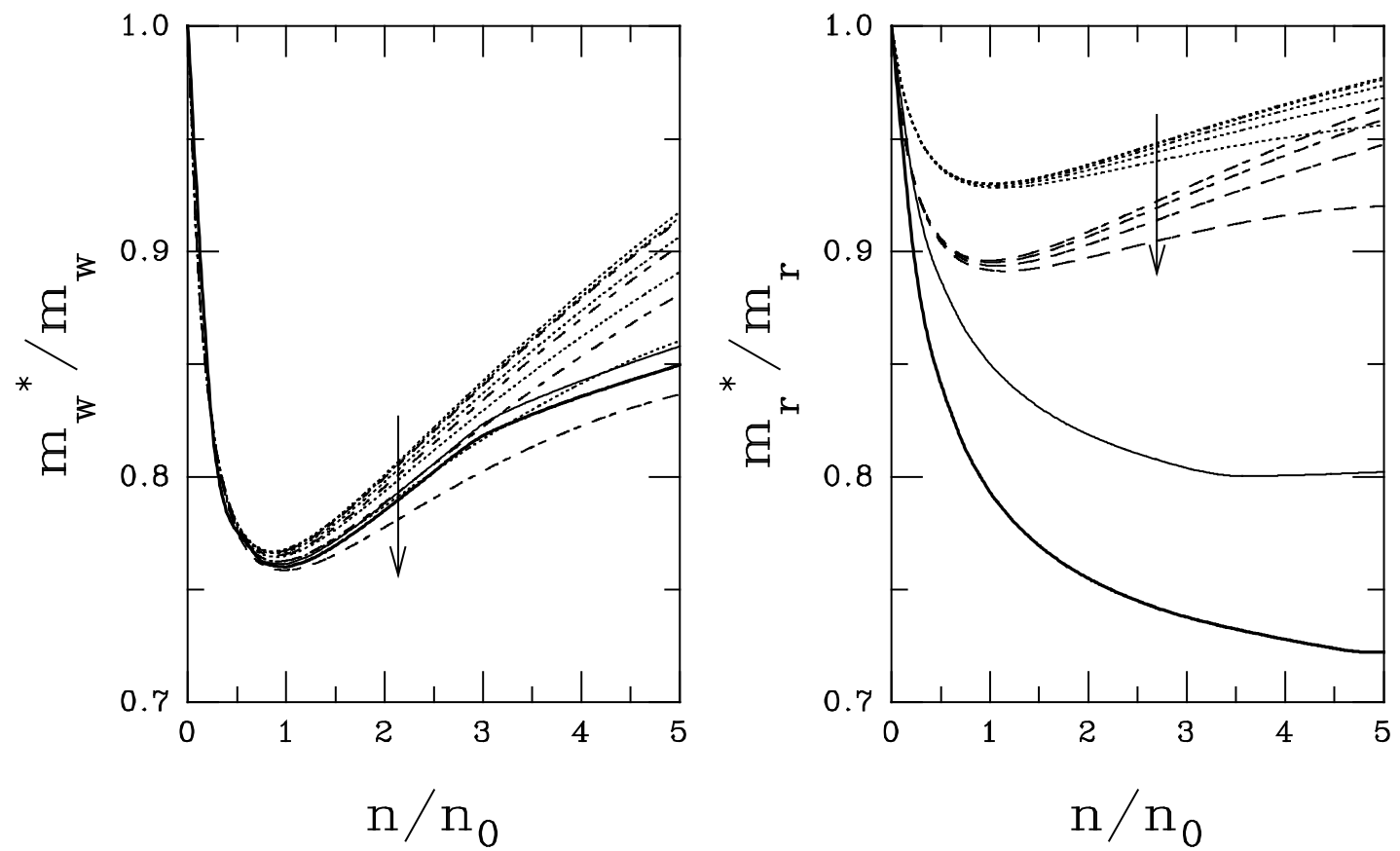

FIG. 15. The density dependence of the vector mesons. The left panel corresponds to the iso-scalar $\omega$ meson, the right panel to the iso-vector $\rho$ meson. In both cases the bold (thin) solid line corresponds to $\beta$-stable matter with coupling set B (A), and dashed (dotted) lines correspond to asymmetric nuclear matter with $\chi=0,0.25,0.50,0.75$, and 1 using the set B (A). The arrow indicates the direction of growing $\chi$.

In order to compare with other similar works, one can appreciate that at high densities $m_{s}^{*}$ is monotonous decreasing and remains below its vacuum value (see Fig. 13). On the contrary, the results found in QHD-I model calculations for symmetric nuclear matter give increasing values of it for $n \geq n_{0}$ [36]. Meanwhile, the behavior of $m_{w}^{*}$ is more alike to ours. The inclusion of polynomic selfinteractions of the $\sigma$ and $\omega$ fields [37] produces increasing or fairly constant density dependence for $m_{w}^{*}$, but if the direct $\sigma-\omega$ interaction is omitted it results in a strongly decreasing behavior [37]. On the other hand, the $\rho$ meson mass can be compared with the results shown in [38]. In both cases $m_{r}^{*}$ is below its vacuum value, for instance at the saturation density $m_{r}^{*}$ is about $10 \%$ down in our calculations against a decrease of $30-40 \%$ in [38]. The differences can be surely assigned to the absence of tensor couplings in our model.

A comparison with the DSCM calculations of [15] shows that the main effect of generalizing the nonlinear $\sigma$ interaction to the vector meson couplings is noteworthy for the effective $\sigma$ and $\omega$ meson masses. Their density dependence is more abrupt in the present work, and generally speaking the values obtained here are lower than in Ref [15]. 


\section{DISCUSSION AND SUMMARY}

In this paper we have proposed an effective relativistic hadronic model inspired in the DSCM to investigate in-medium hadronic properties, in terms of the baryon isospin asymmetry. The non-linear $\sigma$-nucleon interaction is generalized to the isoscalar vector, isovector scalar, and isovector vector channels. Effective medium dependent couplings arise at the MFA, and a residual interaction with one and two meson exchange is obtained beyond the MFA. The equation of state (EoS) for symmetric nuclear matter is softer than the one corresponding to the DSCM. The symmetry energy coefficient shows an intermediate behavior between the QHD model and non-relativistic variational calculations. The asymmetry dependence of the EoS becomes relevant for densities $n \geq 3 n_{0}$, and it is emphasized by the contribution of the $a_{0}(980)$ meson exchange. Temperature effects in the range $0<T<100 \mathrm{MeV}$ are noticeable in the EoS, but moderate in the effective baryon masses. As a particular manifestation of asymmetric matter we study hadronic matter with hyperons, in equilibrium against electroweak decay at $\mathrm{T}=0$. The $\delta$ coupling is the cause of notable modifications in the population of hyperons at high densities. Due to the lower hyperon-meson couplings, relative to the nucleon-meson ones, the hyperonic effective masses decrease more moderately as the baryon density increases.

The effective meson masses have been evaluated at $T=0$ in the RRPA, including particle-antiparticle finite contributions. The regularization procedure left undefined parameters $\lambda_{s}$ and $\lambda_{d}$. We have selected numerical values for them, which differ by one order of magnitude, and are representative of the possible dynamical regimes. In asymmetric nuclear matter the scalar $\sigma$ and $\delta$ mesons exhibit monotonous decreasing masses for high densities, whereas the vector $\omega$ - and $\rho$-meson masses show a slight increase for $n / n_{0}>1$. In all cases the effective masses remain below its vacuum values at extreme densities. The dependence on the asymmetry $\chi$ is more evident for the vector mesons. In $\beta$-stable hadronic matter the density variation of the effective masses of all the mesons considered is damped, becoming approximately constants at twice the saturation density. This effect is due to the particle-antiparticle terms, contributing even for particles out of their Fermi shell.

\section{ACKNOWLEDGMENTS}

This work was partially supported by the CONICET, Argentina.

[1] R. Brockmann and H. Toki, Phys. Rev. Lett. 68, 3408 (1992).

G.Q. Li, R. Machleidt, and Y.Z. Zhuo, Phys. Rev. C 48, 1062 (1993).

[2] S. Haddad and M.K. Weigel, Phys. Rev. C 48, 2740 (1993).

[3] H. Lenske and C. Fuchs, Phys. Lett. B 345, 355 (1995).

C. Fuchs, H. Lenske, and H.H. Wolter, Phys. Rev. C 52, 3043 (1995)

F. de Jong and H. Lenske, Phys. Rev. C 58, 890 (1998), and references therein.

[4] F. de Jong and H. Lenske, Phys. Rev. C 57, 3099 (1998).

[5] F. Hoffmann, C.M. Keil and H. Lenske, LANL report nucl-th/0007050.

[6] S. Typel and H.H. Wolter, Nucl. Phys. A656, 331 (1999).

[7] M. K. Banerjee, Phys. Rev. C 45, 1359 (1992). M.K. Banerjee and J.A. Tjon, Phys. Rev. C 56, 497 (1997).

[8] A.M. Rakhimov, F.C. Khanna, U.T. Yakhshiev and M.M. Musakhanov, Nucl. Phys. A 643, 383 (1998).

[9] K. Saito and A.W. Thomas, Phys. Lett. B 327, 9 (1994).

[10] B. D. Serot and J.D. Walecka, Int. J. Mod. Phys. E 6, 515 (1997).

[11] G.E. Brown and M. Rho, Phys. Rev. Lett. 66, 2720 (1991).

[12] H. Feldmeier and J. Lindner, Z. Phys. A 34183 (1991).

[13] J. Zimanyi and S.A. Moszkowski, Phys. Rev. C 42, 1416 (1990).

[14] A. Bhattacharyya and S. Raha, Phys. Rev. C 53, 522 (1996).

[15] R. Aguirre, Phys. Rev. C 63, 025206 (2001).

[16] R. Aguirre, A.L. de Paoli and O. Civitarese, Nucl. Phys. A 597, 543 (1996).

[17] B.-A. Li, C.M. Ko, and Z. Ren, Phys. Rev. Lett. 78, 1644 (1997).

[18] B.-A. Li, C.M. Ko, and W. Bauer, Int. J. Mod. Phys. E7, 147 (1998).

B.-A. Li, Phys. Rev. Lett. 85, 4221 (2000).

[19] M. Kutschera and W. Wojcik, Phys. Lett. B 223, 11 (1989).

[20] M. Kutschera, Phys. Lett. B 340, 1 (1994). 
[21] S. Kubis and M.Kutschera, Phys. Lett. B 399, 191 (1997).

[22] M. Kutschera and J. Niemiec, Phys. Rev. C 62, 025802 (2000).

[23] C.-H. Lee, T.T.S. Kuo, G.Q. Li, and G.E. Brown, Phys. Rev. C 57, 3488 (1998).

[24] N. Frohlich and H. Baier, Phys. Rev. C 57, 3447 (1998). H. Huber, F. Weber, and M.K. Weigel, Phys. Rev. C 57, 3484 (1998). W. Zuo, I. Bombaci, and U. Lombardo, Phys. Rev. C 60, 024605 (1999).

[25] V. Greco, M. Colonna, M. Di Toro, G. Fabbri, and F. Matera, LANL report nucl-th/0011036.

[26] J.M. Lattimer, C.J. Pethick, M. Prakash, and P. Haensel, Phys. Rev. Lett. 66, 2701 (1991).

[27] M. Prakash, T.L. Ainsworth, and J.M. Lattimer, Phys. Rev. Lett. 61, 2518 (1988).

[28] J.-K. Zhang and D.S. Onley, Phys. Rev. C 44, 2230 (1991). M.M. Sharma, S.A. Moszkowski and P. Ring, Phys. Rev. C 44, 2493 (1991). K. Miyazaki, Prog. Theor. Phys 91, 1271 (1994); ibid. 93, 137 (1995). R.J. Lombard, S. Marcos and J. Mares, Phys. Rev C 51, 1784 (1995).

P. Bernardos, R.J. Lombard, M. Lopez-Quelle, S. Marcos, and R. Niembro, Phys. Rev. C 62, 024314 (2000).

[29] N. K. Glendenning, F. Weber, and S. A. Moszkowski, Phys. Rev. C 45, 844 (1992).

M. Prakash, J.R. Cooke, and J.M. Lattimer, Phys. Rev. D 52, 661 (1995).

[30] S.K. Choudhury and R. Rakshit, Phys. Rev. C 48, 598 (1993).

[31] M. Barranco, R.J. Lombard, S. Marcos, and S.A. Moszkowski, Phys. Rev. C 44, 178 (1991).

[32] R. Aguirre and M. Schvellinger, Phys. Lett. B 400, 245 (1999)

[33] T.S. Biro and J. Zimanyi, Phys. Lett. B 391, 1 (1997).

[34] B. D. Serot and J. D. Walecka, Nucl. Phys A 663, 513 (2000). R. J. Furnstahl and B. D. Serot, Nucl. Phys A 671, 447 (2000).

[35] R.B. Wiringa, V. Fiks, and A. Fabrocini, Phys. Rev. C 38, 1010 (1988).

[36] K. Saito, T. Maruyama, and K. Soutome, Phys. Rev. C 40, 407 (1989).

[37] J.C. Caillon and J. Labarsouque, Phys. Rev. C 62, 035201 (2000).

[38] H. Shiomi and T. Hatsuda, Phys. Lett. B 334, 281 (1994). 